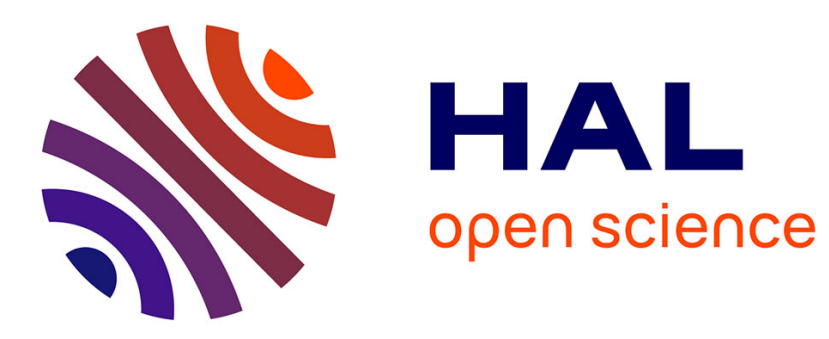

\title{
Identification of Elastic Properties Based on Belief Function Inference
}

\author{
Liqi Sui, Pierre Feissel, Thierry Denoeux
}

\section{To cite this version:}

Liqi Sui, Pierre Feissel, Thierry Denoeux. Identification of Elastic Properties Based on Belief Function Inference. Fourth International Conference Belief Functions: Theory and Applications (BELIEF 2016), Sep 2016, Prague, Czech Republic. pp.182-189, 10.1007/978-3-319-45559-4_19 . hal01397977

\section{HAL Id: hal-01397977 \\ https://hal.science/hal-01397977}

Submitted on 16 Nov 2016

HAL is a multi-disciplinary open access archive for the deposit and dissemination of scientific research documents, whether they are published or not. The documents may come from teaching and research institutions in France or abroad, or from public or private research centers.
L'archive ouverte pluridisciplinaire HAL, est destinée au dépôt et à la diffusion de documents scientifiques de niveau recherche, publiés ou non, émanant des établissements d'enseignement et de recherche français ou étrangers, des laboratoires publics ou privés. 


\title{
Identification of Elastic Properties Based on Belief Function Inference
}

\author{
Liqi Sui ${ }^{1}$, Pierre Feissel ${ }^{1}$, and Thierry Denœux ${ }^{2}$ \\ ${ }^{1}$ UMR CNRS 7337, Laboratoire Roberval, Université de Technologie de Compiègne, \\ Sorbonne Universités, 60203 Compiègne, France \\ ${ }^{2}$ UMR CNRS 7253, Laboratoire Heudiasyc, Université de Technologie de Compiègne, \\ Sorbonne Universités, 60203 Compiègne, France \\ \{liqi.sui, pierre.feissel, thierry.denoeux\}@utc.fr
}

\begin{abstract}
In this paper, we consider parameter identification from measurement fields in an uncertain environment. An approach based on the theory of belief functions is developed to take into account all possible sources of information. Information from measurements is described through likelihood-based belief functions, while consonant random sets are used to handle prior information on the model parameters. Next, we construct the posterior random set by combining measurement and prior information using Dempster's rule. To summarize the posterior random sets, we propose to find the minimal-area region in the parameter space, whose belief and plausibility values exceed given thresholds. This approach was applied to identify the elastic properties of a $2 \mathrm{D}$ plate from a measured kinematic field.
\end{abstract}

Keywords: Identification, measurement field, prior information, aleatory uncertainty, epistemic uncertainty, likelihood-based belief function, belief function theory

\section{Introduction}

In recent years, full field measurements (e.g., kinematic fields) have been increasingly used for the characterization of the mechanical behavior of materials and structures. They allow one to tackle the challenge of identification from heterogeneous tests thanks to their very rich information contents. However, the measurements are always uncertain and the identification problems can be ill-posed. A way to solve this problem is to take advantage of available prior information. Nevertheless, similarly to measurement information, prior information is also tainted with uncertainty. Furthermore, measurement uncertainty and prior information uncertainty have different natures. Measurement uncertainty can be considered as aleatory, whereas prior information uncertainty is epistemic [1] [2]. The uncertainty on prior information can be represented by various approaches, such as intervals [3], possibility theory[4], or imprecise probability[5]. We aim at proposing a unified framework to describe all uncertainties, and a strategy to propagate them. 
This paper focuses on developing a method to identify material parameters from kinematic fields. There are two challenges: firstly, taking into account both measurement and prior information; secondly, quantifying the different kinds of uncertainty and propagating them through models. Belief function theory, also referred to as Evidence theory [6] [7] [8], offers a suitable framework to encode and quantify both epistemic and aleatory uncertainty. Moreover it includes a comprehensive information merging mechanism for combination and conditioning. Some previous studies using belief function theory have focused on the conversion of available information and the propagation of uncertainty through mechanical models [9] [10]. However, very few studies have been devoted to handling uncertainty in identification based on belief functions. In this paper, we explore the possibility of using belief functions theory to quantify uncertainty in identification.

\section{Identification strategy}

We consider the identification of elastic parameters of a $2 \mathrm{D}$ body (plain stress) under loading based on displacement field data. The body shown in Figure 1 is considered as a $2 \mathrm{D}$ domain $\Omega$. The main unknowns are the material parameters collected in $\boldsymbol{\theta}$; the stress field $\boldsymbol{\sigma}$ and the displacement field $\boldsymbol{u}$ are secondary unknowns.

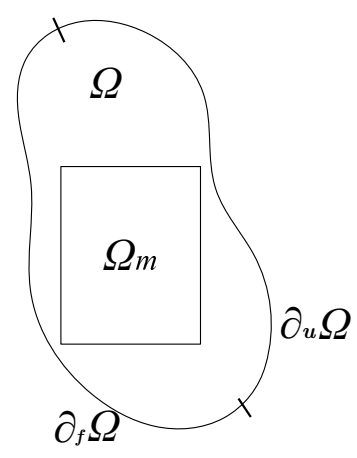

Fig. 1. Mechanical model for identification.

We sum up the equation corresponding to the available information as follows:

- On $\Omega$ :

$$
\begin{array}{ll}
\text { Equilibrium: } & \operatorname{div} \boldsymbol{\sigma}=\mathbf{0}, \\
\text { Constitutive equation: } & \boldsymbol{\sigma}=\boldsymbol{C}(\boldsymbol{\theta}) \boldsymbol{\varepsilon} \text { with } \boldsymbol{\varepsilon}=\frac{1}{2}\left(\nabla \boldsymbol{u}+\nabla^{T} \boldsymbol{u}\right),
\end{array}
$$

where $\boldsymbol{u}, \boldsymbol{\varepsilon}$ and $\boldsymbol{\sigma}$ are, respectively, the displacement, strain and stress fields; 
- On $\partial_{u} \Omega: \boldsymbol{u}=\boldsymbol{u}_{d}$, where $\boldsymbol{u}_{d}$ is a known Dirichlet boundary condition;

- On $\partial_{f} \Omega: \boldsymbol{\sigma} \cdot \boldsymbol{n}=\boldsymbol{f}_{s}$, where $\boldsymbol{f}_{s}$ is a known Neumann boundary condition;

- On $\Omega_{m}: \boldsymbol{u}=\widetilde{\boldsymbol{u}}$. The displacement is measured in $\Omega_{m} \subset \Omega$.

We consider the simple case where $\partial_{u} \Omega \cup \partial_{f} \Omega=\partial \Omega$ and $\partial_{u} \Omega \cap \partial_{f} \Omega=\emptyset$. The purpose of the identification is to find the elastic parameters $\boldsymbol{\theta}$ that are compatible with the above equations, taking into account uncertainty. The available information can be split into three categories:

- Theoretical information, which is considered as reliable and deterministic. We substitute Eq. (2) into Eq. (1) and obtain an implicit function about $\boldsymbol{u}$ and $\boldsymbol{\theta}: \boldsymbol{g}(\boldsymbol{u}, \boldsymbol{\theta})=\mathbf{0}$. Considering the boundary conditions, a well-posed direct problem, whose solution is denoted $\boldsymbol{u}=\boldsymbol{u}(\boldsymbol{\theta})$ for any given $\boldsymbol{\theta}$, can then be defined.

- Experimental information, with mainly aleatory uncertainty. Hence the displacement measurement can be written as

$$
\widetilde{\boldsymbol{u}}=\boldsymbol{u}(\boldsymbol{\theta})+e
$$

where $\boldsymbol{e}$ is the measurement error.

- Background information corresponding to prior knowledge on $\boldsymbol{\theta}$; it is tainted with epistemic uncertainty.

In the following, we will use belief functions and random sets to model uncertainty. Considering a probability space $\left(\Omega, \sigma_{\Omega}, P\right)$, a non-empty set $\boldsymbol{\Theta}$, its power set $2^{\Theta}$ and a strongly measurable multi-valued mapping $\Gamma: \Omega \longrightarrow 2^{\Theta}, \Gamma$ is a random set. For all $\boldsymbol{A} \subseteq \boldsymbol{\Theta}$, the uncertainty of the proposition $\boldsymbol{\theta} \in \boldsymbol{A}$ can be quantified by belief and plausibility function [6] [11]:

$$
\begin{aligned}
\operatorname{Bel}(\boldsymbol{A}) & =P(\{\omega \in \Omega \mid \Gamma(\omega) \subseteq \boldsymbol{A}, \Gamma(\omega) \neq \emptyset\}), \\
\operatorname{Pl}(\boldsymbol{A}) & =P(\{\omega \in \Omega \mid \Gamma(\omega) \cap \boldsymbol{A} \neq \emptyset\}) .
\end{aligned}
$$

$\operatorname{Bel}(\boldsymbol{A})$ is interpreted as the degree of support in the proposition $\boldsymbol{\theta} \in \boldsymbol{A}$, while $\operatorname{Pl}(\boldsymbol{A})$ measures the lack of support in the proposition $\boldsymbol{\theta} \notin \boldsymbol{A}$. The contour function $p l: \Theta \rightarrow[0,1]$ is defined as

$$
p l(\boldsymbol{\theta})=\operatorname{Pl}(\{\boldsymbol{\theta}\}) \text { for all } \boldsymbol{\theta} \in \boldsymbol{\Theta} .
$$

\subsection{Measurement information}

The measurement $\widetilde{\boldsymbol{u}}$ is assumed to be known with some aleatory uncertainty (see Eq. (3)). The error is assumed to be a random Gaussian noise, with known covariance $\boldsymbol{e} \sim \mathcal{N}(\mathbf{0}, \boldsymbol{D})$. Based on [6] [8], the uncertainty about $\boldsymbol{\theta}$ is represented by a consonant likelihood-based belief function, whose contour function equals the normalized likelihood function:

$$
p l(\boldsymbol{\theta} ; \widetilde{\boldsymbol{u}})=\frac{L(\boldsymbol{\theta} ; \widetilde{\boldsymbol{u}})}{\sup _{\boldsymbol{\theta}} L(\boldsymbol{\theta} ; \widetilde{\boldsymbol{u}})},
$$


where $L(\boldsymbol{\theta} ; \widetilde{\boldsymbol{u}})$ is the likelihood function,

$$
L(\boldsymbol{\theta} ; \widetilde{\boldsymbol{u}})=(2 \pi \operatorname{det} \boldsymbol{D})^{-1 / 2} \exp \left[-\frac{1}{2}(\boldsymbol{u}(\boldsymbol{\theta})-\widetilde{\boldsymbol{u}})^{T} \boldsymbol{D}^{-1}(\boldsymbol{u}(\boldsymbol{\theta})-\widetilde{\boldsymbol{u}})\right] .
$$

The contour function $p l(\boldsymbol{\theta} ; \widetilde{\boldsymbol{u}})$ in Eq. (7) is normalized. It is equivalent to a possibility distribution, and corresponds to a consonant random set.

\subsection{Prior information}

Prior information with epistemic uncertainty is represented by a possibility distribution $\pi(\boldsymbol{\theta})$, which induces the consonant random set

$$
\Gamma(\omega)=\{\boldsymbol{\theta} \in \boldsymbol{\Theta} \mid \pi(\boldsymbol{\theta}) \geq \omega\} .
$$

The consonant random set shown in Fig. 2 expresses an expert opinion about $\boldsymbol{\theta}$ : values of $\boldsymbol{\theta}$ outside interval $[a, d]$ are considered as impossible, while values inside interval $[b, c]$ are considered as fully possible. Indeed, $\Gamma(\omega) \subseteq[a, d]$ and $[b, c] \subseteq \Gamma(\omega)$ for any $\omega \in[0,1]$. In this paper, we assume that $\omega \sim \mathcal{U}[0,1]$.

Moreover, in the framework of belief function theory, the discounting operation allows us to express the degree of confidence in a source of information. Assume, for instance, that an expert uses the possibility distribution of Fig. 2 to represent their opinion about a parameter, and we have a degree of confidence $m_{\boldsymbol{\Theta}} \in[0,1]$ in this opinion. We can then assign the value $m_{\boldsymbol{\Theta}}$ to the whole set $\boldsymbol{\Theta}$, i.e., $P(\{\omega \in \Omega \mid \Gamma(\omega)=\boldsymbol{\Theta}\})=m_{\boldsymbol{\Theta}}$. When $m_{\boldsymbol{\Theta}}=0$, we fully trust the expert's opinion; when $m_{\Theta}=1$, we totally doubt it. The discounted possibility distribution is shown in Fig. 3. We notice that it has an infinite support.

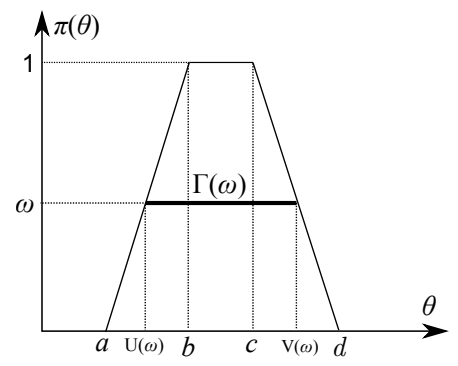

Fig. 2. Possibility distribution

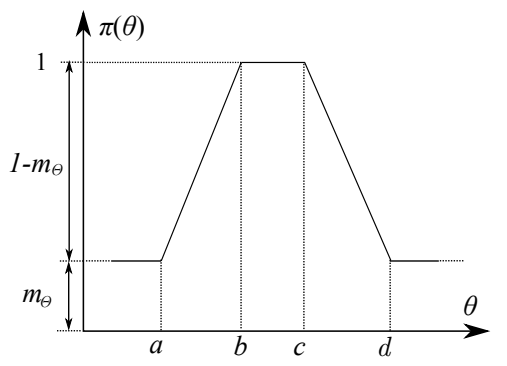

Fig. 3. Discounted Possibility distribution

\subsection{Dempster's rule}

Measurement and prior information typically induce two random sets represented by possibility distributions. Aggregation of information from multiple 
sources is an important step in the modeling of uncertainty. Dempster's rule [6] is a combination mechanism in belief function theory. This rule is often used to combine belief functions defined on finite sets. Here, we reformulate it in the infinite setting. Consider two random sets $\left(\Omega_{k}, \sigma_{\Omega_{k}}, P_{k}, \Gamma_{k}\right), k=1,2$. Let $\Omega=\Omega_{1} \times \Omega_{2}$ be the product space, $P=P_{1} \otimes P_{2}$ the product measure on $\sigma_{\Omega}=\sigma_{\Omega_{1}} \otimes \sigma_{\Omega_{2}}$, and $\Gamma_{p}$ the multi-valued mapping defined by: $\forall\left(\omega_{1}, \omega_{2}\right) \in \Omega_{p}$, $\Gamma_{p}\left(\omega_{1}, \omega_{2}\right)=\Gamma_{1}\left(\omega_{1}\right) \cap \Gamma_{2}\left(\omega_{2}\right)$. The combined random set is $\left(\Omega, \sigma_{\Omega}, P, \Gamma_{p}\right)$. It induces the following belief and plausibility functions: for any $\boldsymbol{A} \subset \boldsymbol{\Theta}$,

$$
\begin{aligned}
\operatorname{Bel}(\boldsymbol{A}) & =\frac{P\left(\left\{\left(\omega_{1}, \omega_{2}\right) \in \Omega \mid \Gamma_{p}\left(\omega_{1}, \omega_{2}\right) \subseteq \boldsymbol{A}, \Gamma_{p}\left(\omega_{1}, \omega_{2}\right) \neq \emptyset\right\}\right)}{P\left(\left\{\left(\omega_{1}, \omega_{2}\right) \in \Omega \mid \Gamma_{p}\left(\omega_{1}, \omega_{2}\right) \neq \emptyset\right\}\right)} \\
P l(\boldsymbol{A}) & =\frac{P\left(\left\{\left(\omega_{1}, \omega_{2}\right) \in \Omega \mid \Gamma_{p}\left(\omega_{1}, \omega_{2}\right) \cap \boldsymbol{A} \neq \emptyset\right\}\right)}{P\left(\left\{\left(\omega_{1}, \omega_{2}\right) \in \Omega \mid \Gamma_{p}\left(\omega_{1}, \omega_{2}\right) \neq \emptyset\right\}\right)}
\end{aligned}
$$

The degree of conflict is:

$$
k=P\left(\left\{\left(\omega_{1}, \omega_{2}\right) \in \Omega \mid \Gamma_{p}\left(\omega_{1}, \omega_{2}\right)=\emptyset\right\}\right) .
$$

It is a measure of the compatibility between the two sources of information.

\section{Numerical implementation and posterior exploration}

In this paper, we use Monte Carlo simulation to implement Dempster's rule for combining information. Considering two possibility distributions $\pi_{1}(\boldsymbol{\theta})$ and $\pi_{2}(\boldsymbol{\theta})$, we can draw $\left(\omega_{1}, \omega_{2}\right)$ uniformly from $[0,1]^{2}$ and cut the two possibility distributions, respectively, at levels $\omega_{1}$ and $\omega_{2}$. Then we intersect the two $\omega$ level cuts. After iterating the above process a large number of times, we obtain a collection of subsets or domains with irregular shapes. Therefore, we need a strategy to describe domains of arbitrary shape. Here, we propose to describe a subset using a cloud of points generated by a Halton sequence [12]. For each domain, the value at any point is 1 if the point is inside this domain, and 0 otherwise. Such a description is suitable for Boolean operations as required by Dempster's rule.

After merging information, we obtain a collection of posterior subsets that need to be exploited. The contour function $p l(\boldsymbol{\theta})$ is an easy and direct way to summarize information. The greatest $p l(\boldsymbol{\theta})$ value corresponds to the most possible $\boldsymbol{\theta}$. Consequently Eq. (13) can serve as a point identification method:

$$
\widehat{\boldsymbol{\theta}}=\arg \max p l(\boldsymbol{\theta}) .
$$

For further exploitation of posterior random sets, we search for a minimal subset $\boldsymbol{R} \subseteq \boldsymbol{\Theta}$ such that $\operatorname{Pl}(\boldsymbol{R})$ and $\operatorname{Bel}(\boldsymbol{R})$ are larger than threshold values $\delta_{P l}$ and $\delta_{B e l}$. Formally, we need to solve the following constrained minimization problem:

$$
\widehat{\boldsymbol{R}}=\arg \min _{\boldsymbol{R} \subseteq \boldsymbol{\Theta}} V(\boldsymbol{R}),
$$


such that

$$
\left\{\begin{array}{l}
P l(\boldsymbol{R}) \geq \delta_{P l} \\
\operatorname{Bel}(\boldsymbol{R}) \geq \delta_{B e l}
\end{array},\right.
$$

where $V(\boldsymbol{R})$ is the area or volume of $\boldsymbol{R}$.

\section{Application}

In this section, we present as a numerical example the identification of the Lamé coefficients $\boldsymbol{\theta}=\{\lambda, \mu\}$ describing the elastic properties of a $2 \mathrm{D}$ plate from a tensile test. The measurement is the displacement field on the whole plate and the applied traction. It was created based on a reference finite element calculation with reference value $\{\lambda, \mu\}=\{1,1\}$ and adding a $5 \%$ Gaussian noise to represent the measurement error.

The prior information, which comes from expert opinions, was expressed by possibility distributions. In order to check the performance of this approach, two scenarios were considered: in the first scenario, the expert possibility distributions are close to the reference values; in the second scenario, they are far from the reference values. We assumed a $80 \%$ degree of confidence in the expert opinions in both scenarios. The possibility distributions considered in both scenarios are shown in Fig. 4.

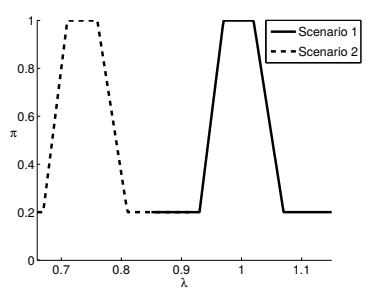

(a)

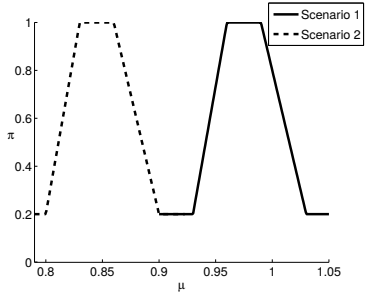

(b)

Fig. 4. Prior information on $\lambda$ (a) and $\mu$ (b).

Based on the discussion in Section 2.3, we used Dempster's rule to combine the information. After computing the $\omega$-level cuts of the two distributions and intersecting the random sets, we obtained a posterior random set. The degrees of conflict in scenarios 1 and 2 were, respectively, $\widehat{k}_{1}=0.3867$ and $\widehat{k}_{2}=0.7996$. If the degree of conflict $k$ is too large, at least one source is likely to provide wrong information.

The estimator $\widehat{p l}(\boldsymbol{\theta})$ of the contour function is shown in Fig. 5. The value $\boldsymbol{\theta}_{\max }$ with maximum plausibility can be used as a point estimator. For scenario 1, we obtained $\boldsymbol{\theta}_{\max }=\{0.943,0.939\}$; for scenario $2, \boldsymbol{\theta}_{\max }=\{0.935,0.937\}$. Thanks to the discounting operation, the contour function keeps the same form as the 


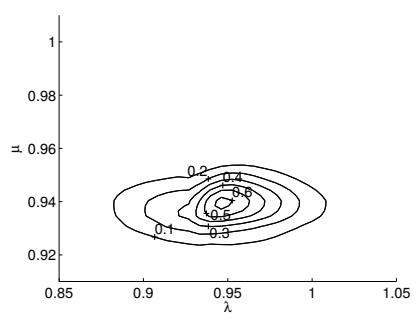

(a)

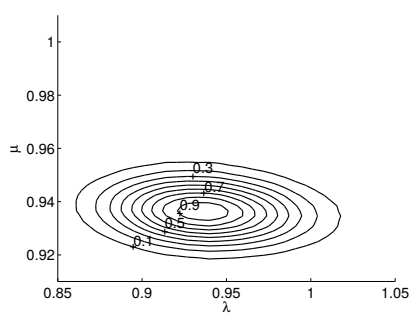

(b)

Fig. 5. Contour function $\widehat{p l}(\boldsymbol{\theta})$ : (a) scenarios 1; (b) scenarios 2 .

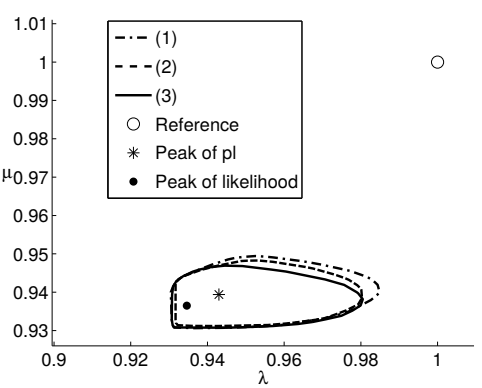

(a)

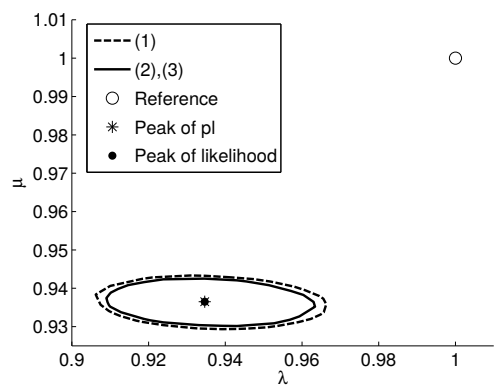

(b)

Fig. 6. Minimal-area domains $\widehat{\boldsymbol{R}}$ : (a) scenario 1 (b) scenario 2 
possibility distribution from the measurement, even when the prior information is inaccurate.

Lastly, we focussed on finding a minimum subset $\boldsymbol{R}$ with $\operatorname{Pl}(\boldsymbol{R})$ and $\operatorname{Bel}(\boldsymbol{R})$ larger than given threshold values $\delta_{B e l}$ and $\delta_{P l}$. We set (1) $\delta_{B e l}=0.30$ and $\delta_{P l}=0.95 ;(2) \delta_{B e l}=0.25$ and $\delta_{P l}=0.95(3) \delta_{B e l}=0.25$ and $\delta_{P l}=0.90$. The obtained subsets $\widehat{\boldsymbol{R}}$ for the two scenarios are shown in Fig. 6 . It is clear that the area of minimum subsets grows as thresholds $\delta_{B e l}$ and $\delta_{P l}$ increase. As compared to those in scenario 2, the minimal subsets in scenario 1 move to top right because of the influence of prior information. In scenario 2 , the prior information does not affect the measurement information; consequently, the minimal subsets reflect the form of the likelihood function.

\section{Conclusion}

In this paper, we have presented an identification strategy based on belief function theory. This approach allows for the representation and combination of prior and measurement information. Point clouds were used to describe multidimension random sets. Dempster's rule was used to combine random sets from prior and measurement information. Finally, posterior random sets were obtained and explored. This approach makes it possible to encode and propagate epistemic and aleatory uncertainty in a unified framework. The discounting operation allows us to take into account the reliability of the sources of information; as a result, inaccurate information only marginally affects measurement information. The two functions $\mathrm{Bel}$ and $\mathrm{Pl}$ provide a rich description of the uncertainty on model parameters, taking into account prior information. In the future, our research will focus on accounting for uncertainty in mechanical models and on the application of this approach to more complex structures.

Acknowledgments. This research was supported by the Labex MS2T, which was funded by the French Government, through the program "Investments for the future" by the National Agency for Research (reference ANR-11-IDEX-0004$02)$.

\section{References}

1. Scott Ferson and Lev R Ginzburg. Different methods are needed to propagate ignorance and variability. Reliability Engineering 85 System Safety, 54(2):133-144, 1996.

2. Jon C Helton and David E Burmaster. Guest editorial: treatment of aleatory and epistemic uncertainty in performance assessments for complex systems. Reliability Engineering 85 System Safety, 54(2):91-94, 1996.

3. Ramon E Moore. Interval analysis, volume 4. Prentice-Hall Englewood Cliffs, 1966.

4. Didier Dubois, Etienne Kerre, Radko Mesiar, and Henri Prade. Fuzzy interval analysis. In Fundamentals of fuzzy sets, pages 483-581. Springer, 2000. 
5. Scott Ferson, Vladik Kreinovich, Lev Ginzburg, Davis S Myers, and Kari Sentz. Constructing probability boxes and Dempster-Shafer structures, volume 835. Sandia National Laboratories, 2002.

6. Glenn Shafer. A mathematical theory of evidence, volume 1. Princeton university press Princeton, 1976.

7. Philippe Smets. Belief functions on real numbers. International journal of approximate reasoning, 40(3):181-223, 2005.

8. Thierry Denoeux. Likelihood-based belief function: Justification and some extensions to low-quality data. International Journal of Approximate Reasoning, 55(7):1535-1547, 2014.

9. Fulvio Tonon. Using random set theory to propagate epistemic uncertainty through a mechanical system. Reliability Engineering \& System Safety, 85(1):169-181, 2004.

10. Fulvio Tonon, Ha-Rok Bae, Ramana V Grandhi, and Chris L Pettit. Using random set theory to calculate reliability bounds for a wing structure. Structures and Infrastructure Engineering, 2(3-4):191-200, 2006.

11. Glenn Shafer. Allocations of probability. The Annals of Probability, pages 827-839, 1979.

12. Ladislav Kocis and William J Whiten. Computational investigations of lowdiscrepancy sequences. ACM Transactions on Mathematical Software (TOMS), 23(2):266-294, 1997. 